\title{
Roland Barthes par Roland Barthes ou Le démon de la totalité
}

\section{Claude Coste}

\section{OpenEdition}

12 Journals

Édition électronique

URL : http://journals.openedition.org/recherchestravaux/372

DOI : 10.4000/recherchestravaux.372

ISSN : 1969-6434

Éditeur

UGA Éditions/Université Grenoble Alpes

\section{Édition imprimée}

Date de publication : 30 décembre 2009

Pagination : 35-54

ISBN : 978-2-84310-159-5

ISSN : 0151-1874

\section{Référence électronique}

Claude Coste, "Roland Barthes par Roland Barthes ou Le démon de la totalité », Recherches \& Travaux [En ligne], 75 | 2009, mis en ligne le 30 juin 2011, consulté le 10 décembre 2020. URL : http://

journals.openedition.org/recherchestravaux/372; DOI : https://doi.org/10.4000/recherchestravaux. 372 


\section{Roland Barthes par Roland Barthes ou Le démon de la totalité}

\section{Du fragment à la totalité}

Quand il paraît en 1975, Roland Barthes par Roland Barthes fonde cette lignée d'autoportraits qui connaîtra un tel développement dans la littérature française contemporaineI. Par l'étoilement d'une centaine de fragments classés selon l'ordre alphabétique, Barthes évoque principalement son œuvre et sa vie intellectuelle, non sans ménager une place importante à son enfance et à quelques traits plus intimes. Fini le temps des Mémoires d'outre-tombe ou même de l'autobiographie plus ironique, mais très composée, que représentent Si le grain ne meurt de Gide ou Les Mots de Sartre. C'est désormais le fragment qui devient l'outil le plus efficace pour chercher à se dire au public des lecteurs. Mais comment faut-il entendre le mot «fragment» dont Barthes fait un usage récurrent et qu'il confond souvent avec «forme brève» et "écriture discontinue»? Pour en rester à une appréhension simple, on dira que tout fragment entre nécessairement en relation avec une forme de totalité, mais que cette totalité est absente (ce qui distingue le fragment de la simple partie). Héraclite ou Pascal n'ont pas écrit de fragments : c'est la vie ou l'histoire qui se sont chargées de compromettre l'intégrité de l'œuvre, perdue chez le premier, jamais atteinte par le second. Sans lien avec les injures du temps, la fragmentation relève aussi d'un geste de l'auteur. De subi, le fragment devient alors le produit d'une volonté créatrice qui construit une sorte

I. Roland Barthes par Roland Barthes [1975] (Désormais abrégé : RB), Seuil, «Écrivains de toujours", I995. 
de simulacre, sans cesser de penser l'œuvre en relation plus ou moins étroite avec une idée de totalité. Qu'elle soit perdue (la plénitude du verbe divin selon Derrida), qu'elle soit à venir (le rêve mallarméen du Livre) ou qu'elle soit tout bonnement refusée (pour des raisons qui restent à éclaircir), la totalité est nécessaire pour faire exister le fragment comme fragment - et le distinguer de la citation, de l'extrait ou de la simple forme brève (la maxime existe par et pour elle-même, comme totalité ou comme partie d'un recueil).

De ces trois formes de totalité absente, qui, d'ailleurs, ne s'excluent pas l'une l'autre, on trouverait les traces dans le texte de Barthes. Mais c'est incontestablement la troisième, la totalité refusée, qui s'impose avec la plus grande évidence. En effet, qu'est-ce que le fragment pour Barthes sinon le moyen de manifester son rejet de la totalité formelle, rhétorique, c'est-à-dire de toutes ces écritures du continu que sont le récit, le traité ou la dissertation? Réfléchissant à sa propre poétique, Barthes exprime clairement ce refus d'une totalité factice, illusoire, et préfère le fragment, selon le mot d'ordre gidien, "parce que l'incohérence est préférable à l'ordre qui déforme» ("Le cercle des fragments ", $R B$, p. 89. Une fois encore, l'ennemi, c'est l'«Imaginaire», notion qu'il faut prendre dans un sens très large, à mi-chemin de La Rochefoucauld et de Lacan, et que l'on pourrait définir comme la représentation illusoire de soi-même (et au-delà comme toute forme de représentation se donnant pour la réalité). Proche de la bêtise flaubertienne ou de la mauvaise foi sartrienne, l'Imaginaire apporte au sujet le repos d'une identité figée, le confort d'une totalité sur mesure - auquel l'écriture fragmentaire oppose sa puissance de dénonciation (l'éclat récuse le portrait) et d'esquive (le discontinu retarde le retour inévitable de l'Imaginaire). En d'autres termes, quand tant d'autres créateurs chantent la mélancolie du fragment, la nostalgie ou l'espoir de la totalité perdue, Barthes cultive une forme d'écriture qui, ici et maintenant, garantit le double bonheur de la surprise et de la liberté. L'affaire semble donc définitivement close...

Pourtant, malgré les lieux communs de la critique, malgré les déclarations les plus claires de l'auteur, Barthes n'a jamais renoncé à la totalité... Si l'on considère ses dernières œuvres, le temps de la fragmentation semble même terminé. Le continu rhétorique fait sa réapparition dans La Chambre claire, œuvre qui, loin de l'ordre alphabétique, se présente comme une véritable quête de l'essence photographique dans le sillage évident de la Recherche proustienne. De la même manière, La Préparation du roman ${ }^{2}$, rompant avec l'organisation a-systématique des cours précédents, suit le cheminement

2. R. Barthes, La Préparation du roman I et II, Cours et séminaires au Collège de France (1978-1979 et 1979-1980), Texte établi, annoté et présenté par N. Léger, Seuil/IMEC, "Traces écrites", 2003. 
d'une autre forme de quête : le professeur se met dans la position d'écrire un roman, et ce sont les différentes étapes de cette aventure que les auditeurs ont pu suivre de séance en séance. Mais le démon de la totalité n’obsède pas seulement le tout "dernier Barthes». Dès Roland Barthes par Roland Barthes, en effet, le désir de totalité hante l'œuvre, sans remettre en cause sa structure fragmentaire. Pour assumer pleinement ce paradoxe, faut-il déjà chercher du côté du roman? La phrase introductive («Tout ceci doit être considéré comme dit par un personnage de roman»), les références à Balzac, Flaubert ou Proust pour évoquer son propre «roman familial», installent le genre à l'horizon du texte comme une lointaine puissance tutélaire. Mais à l'horizon seulement : malgré sa séduction, malgré le poids de la fiction et de l'intertextualité, le roman reste encore un contre-modèle, condamné pour ce qui le constitue en essence, le récit.

En 1975, Barthes se tient encore au «seuil du roman». C'est donc ailleurs qu'il convient de chercher le fantôme de la totalité. Venu cette fois-ci de l'œuvre antérieure, le "panorama" s'offrirait-il comme la solution? Tantôt pris au sens propre (dans La Tour Eiffel' ${ }^{3}$ ), tantôt au sens figuré (dans Le Degré zéro de l'écriture), le panorama renvoie chez Barthes à l'ivresse des sommets et des grandes ouvertures. Du haut de la tour Eiffel, la ville de Paris s'offre au regard d'un spectateur qui se laisse griser par l'illusion de "comprendre" le monde, c'est-à-dire de l'embrasser des yeux pour le rendre intelligible. Les premiers chapitres du Degré zéro de l'écriture proposaient déjà une autre forme de "panorama» qu'il faut prendre cette fois-ci d'une manière plus abstraite. Comme on s'en souvient, Barthes se lance dans une vaste reconstitution historique, traçant des lignes droites, ouvrant des perspectives qui permettent d'embrasser tout le $\mathrm{XIX}^{\mathrm{e}}$ siècle et de lui donner forme par la pensée. On n'est pas si loin de la préface de Cromwell ou des Mots et les choses: Barthes comme Hugo ou Foucault s'abandonne à l'ivresse de l'intelligibilité, même si l'on apprend des horreurs sur la mort de l'homme ou la fin des civilisations. Pour accompagner cette vue cavalière, cette trouée heureuse dans le massif de l'histoire, le mot «euphorie» revient de façon récurrente dans Le Degré zéro de l'écriture : il apporte une tonalité positive dans la quête impossible de cette écriture non marquée, de cette écriture blanche qui passerait pour l'Immaculée Conception de la littérature.

Que reste-t-il de cette euphorie du panorama dans Roland Barthes par Roland Barthes? De toutes ces manifestations, géographiques ou métaphoriques, le livre fragmentaire se contente de recueillir quelques débris, comme dans «Phases» (RB, p. I29) où l'auteur dresse et commente le tableau des

3. La Tour Eiffel [1964] (Désormais abrégé TE), texte de R. Barthes, photographies de A. Martin, Delpire, CNP/Seuil, 1989. 
grands moments et des intertextes de sa carrière. Le ton peut se montrer plus ironique quand il s'agit de jeter un regard rétrospectif sur telle étape du parcours :

Même dans sa phase structurale, où la tâche essentielle était de décrire l'intelligible humain, il a toujours associé l'activité intellectuelle à une jouissance : le panorama, par exemple, - ce qu'on voit de la tour Eiffel (TE, p. 64) - est un objet à la fois intellectif et heureux : il libère le corps dans le moment même où il lui donne l'illusion de "comprendre» le champ de son regard. ("L'idée comme jouissance», $R B$, p. 97)

On retrouve la même ironie, un peu plus amère, dans le fragment «Emploi du temps». Après avoir énuméré tous les événements de sa journée d'écrivain en vacances, Barthes conclut sans ménagement devant un si beau panorama et une si cette belle perspective :

Tout cela n'a aucun intérêt. Bien plus : nous seulement vous marquez votre appartenance de classe, mais encore vous faites de cette marque une confidence littéraire, dont la futilité n'est plus reçue : vous vous constituez fantasmatiquement en "écrivain» ou pire encore : vous vous constituez. («Emploi du temps", $R B$, p. 79)

Bref, du panorama, on tombe dans l'Imaginaire... Quant au sens propre du mot, il se contente d'une petite apparition avec le tramway Bayonne-Biarritz dont le trajet permet de découvrir un beau "panorama» sur le Pays basque («La baladeuse», $R B$, p. 54). Tout cela pèse bien peu, il faut se faire une raison : le panorama a fait son temps et le roman hésite encore à se proposer comme modèle structurel. Consubstantielle au fragment, la totalité dans Roland Barthes par Roland Barthes est donc à chercher ailleurs.

\section{Le sentiment de l'existence}

«Le monstre de la totalité» : c'est sous ce titre presque provocateur que Barthes décide de terminer son autoportrait en éclats. Si le premier paragraphe du fragment correspond à une vision négative - et attendue - de la totalité comme monstre, le second surprend par un tournant de pensée et d'écriture, aussi tardif qu' efficace :

Autre discours : ce 6 août, à la campagne, c'est le matin d'un jour splendide : soleil, chaleur, fleurs, silence, calme, rayonnement. Rien ne rode, ni le désir, ni l'agression; seul le travail est là, devant moi, comme une sorte d'être universel : tout est plein. Ce serait donc cela, la Nature? Une absence... du reste? La Totalité? $\left(R B, \mathrm{p} .156^{4}\right)$

4. Voir également la légende, p. 32 : «Le délice de ces matinées à U. : le soleil, la maison, les roses, le silence, la musique, le café, le travail, la quiétude insexuelle, la vacance des agressions...» 
Quand tout le livre semblait emporté par l'euphorie de l'écriture fragmentaire, prendre congé du lecteur avec le mot «totalité» ne manque pas de surprendre. Dépourvu de valeur conclusive, mais dernière impression laissée par le texte, le fragment final, c'est le moins qu'on puisse dire, ne livre pas immédiatement son sens. Comme souvent chez Barthes, la signification se construit par un jeu d'échos, s'éclaire par les différentes reprises et variations d'un même mot ou d'une même formule. Quelques pages plus haut, une parenthèse, en glosant le «etc.» qui clôt la série des anamnèses ${ }^{5}$, appréhendait déjà la nature comme l'espace par excellence de la suffisance et de la plénitude : «Etc. (N'étant pas de l'ordre de la Nature, l'anamnèse comporte un «etc.».)» ("Pauses : anamnèses», RB, p. IO2). La précision est parfaitement claire : d'un côté les souvenirs qui reviennent sans fin à la conscience, de l'autre, la Nature qui se présente comme une totalité indépassable, comme l'image même de la perfection. Quasi contemporain du livre, le séminaire sur Le Discours amoureux revient, dans un contexte différent, sur la même référence : "(le "etc." compte beaucoup. Rappelons-nous Valéry : dans la nature, il n'y a pas de "etc." Tout ordre qui comporte un "etc." n'est pas dans la Nature, disons le Réel. Etc. : mot de l'Imaginaire) ${ }^{6}$ ». La référence valérienne est suffisamment insistante pour ne pas manquer d'intriguer le lecteur.

D'un texte à l'autre, comme par écho et par diffraction, un nouveau système homologique se met ainsi en place : la Nature s'oppose désormais à l'Imaginaire et la Totalité à l'Incomplétude. Or, pareille équivalence, qui reste encore mystérieuse, vient remettre en cause ou complexifier une relation qui semblait exclusive dans Roland Barthes par Roland Barthes : jusqu'ici, à l'image, globale, totalitaire et mensongère, l'écrivain tentait d'échapper par une double activité de fragmentation et de multiplication. Par la pulvérisation du texte, par le refus de classer les fragments, sinon selon le code alphabétique, Barthes luttait contre la puissance d'une totalité imaginaire qui cherche à imposer l'illusion des essences. Mais voici que sans contredire le chant principal, une autre musique se fait entendre : lancé comme une flèche du Parthe, au moment de fermer le livre, le mot "totalité» donne l'impression que la pensée bascule dans une nouvelle dimension et ouvre une autre relation possible entre la totalité qui opprime et la fragmentation qui sauve. Autrement dit, dans le monde chatoyant de Barthes, une totalité peut en cacher une autre...

Clé manifeste de cette nouvelle attitude, le mot «Nature» recoupe de nombreuses acceptions. Entre la nature comme négation bourgeoise de

5. Comme une pause au milieu du livre, Barthes énumère une série de souvenirs d'enfance.

6. Le Discours amoureux. Séminaire à l'École pratique des hautes études 1974-1976 suivi de Fragments d'un discours amoureux : inédits, présentation et édition de Cl. Coste, Seuil, «Traces écrites", 2007, p. 204. 
l'Histoire et les paysages du Sud-Ouest chers à Barthes 7 , le même mot désigne des réalités si différentes qu'un détour par l'œuvre de Rousseau permettra sans doute d'y voir un peu plus clair. Comme on s'en souvient, l'auteur des Rêveries du promeneur solitaire se lance dans une quête du bonheur dont le contact avec la nature apparaît comme une des voies d'accès privilégiées. Or, ce n'est pas un hasard, Barthes revient souvent dans son œuvre sur ce texte qui manifestement le fascine. Dès I944, dans Existences, la revue des étudiants du sanatorium de Saint-Hilaire-du-Touvet, il publie "Plaisir aux classiques», un texte capital qui, d'une certaine manière et en évitant toute perspective téléologique, vaut comme matrice d'une grande partie de son œuvre à venir. Que lit-on en effet? Après un vif éloge des "classiques", Barthes propose une anthologie de citations, dont un célèbre passage tiré de Rousseau. Tout lycéen français se souvient bien de l'accident de la rue Ménilmontant raconté dans la Seconde Promenade : parti pour herboriser, Rousseau remonte la longue rue parisienne, ne parvient pas à éviter un chien danois déboulant à toute vitesse, s'évanouit après une chute et se réveille peu à peu, reprenant conscience sur un mode euphorique. Voici la citation retenue par Barthes et appelée à un bel avenir dans son œuvre :

La nuit s'avançait. J'aperçus le ciel, quelques étoiles, et un peu de verdure. Cette première sensation fut un moment délicieux. Je ne me sentais encore que par là. Je naissais dans cet instant à la vie; et il me semblait que je remplissais de ma légère existence tous les objets que j'apercevais... ${ }^{8}$

Cette expérience singulière de bonheur revient dans de nombreux autres passages des Rêveries - au bord du lac de Bienne, en particulier, que Rousseau décrit au cours de la non moins célèbre Cinquième promenade (ou devant le feu de Milord Edouard dans La Nouvelle Héloïse). D'où provient cette douce euphorie, à la fois si familière (chacun peut la connaître) et totalement atypique (par sa rareté) ? Heureux de se retrouver au contact de la nature, bercé par le rythme des vagues du lac de Bienne ou étourdi par sa chute, Rousseau découvre, ce qu'on appellera : le sentiment de l'existence. Quand le cogito cartésien repose sur la célèbre formule : "Je pense, donc je suis", le sujet rousseauiste se reconnaitrait plutôt dans un «Je sens, donc je suis» ou,

7. «Commence alors la grande lumière du Sud-Ouest, noble et subtile tout à la fois; jamais grise, jamais basse (même lorsque le soleil ne luit pas), c'est une lumière-espace, définie moins par les couleurs dont elle affecte les choses (comme dans l'autre Midi) que par la qualité éminemment habitable qu'elle donne à la terre. Je ne trouve pas d'autre moyen que de dire : c'est une lumière lumineuse." R. Barthes, "La lumière du Sud-Ouest», Euvres complètes, t. V [1977-1980], nouvelle édition revue, corrigée et présentée par É. Marty, Seuil, 2002, p. 33r.

8. "Plaisir aux classiques", Euvres complètes, t. I (1942-196I) nouvelle édition revue, corrigée et présentée par É. Marty, Seuil, 2002, p. 64. 
mieux encore, dans un «Je sens que je suis». Débarrassé de tous les attributs (Barthes aurait dit les «adjectifs»), la conscience s'allège et le sujet, ignorant tout de lui hors sa pure existence, ne s'appréhende plus que par la sensation d'être. Cette conscience très particulière, qui lie inextricablement le sujet et le monde, mais décante l'un et l'autre de tous les «bruits" parasites, de tous les "etc.», fonde le bonheur sur une plénitude, sur une totalité que le moindre événement de la vie vient, bien sûr, mettre à mal : interrompant l'extase, le réveil est rapide et le bonheur s'enfuit.

À deux siècles de distance, le dernier paragraphe du Roland Barthes par Roland Barthes dialogue implicitement avec le texte de Rousseau et sa douce euphorie. Mais d'autres textes ne sont pas en reste. Les notes de cours sur Le Neutre, habitées par le rêve d'une vie sans histoire, par le désir d'échapper à la tyrannie de l'antithèse et du paradigme, citent à nouveau la description du réveil à Ménilmontant ${ }^{9}$ et consacrent de longues pages à la paix du lac de Bienne, sans faire référence, il est vrai, au "sentiment de l'existence ${ }^{\mathrm{IO}}$ ». De 1944 à 1978 , Barthes se contente de citer ou de décrire. Il faudra attendre un de ses derniers textes, un article publié par le journal Le Monde, pour non seulement retrouver le même passage une fois encore, mais pour lire le long commentaire que Barthes s'était refusé jusqu'alors. Voici l'intégralité de cette analyse, reformulation lointaine à la fois du commentaire de Rousseau et de la fin de Roland Barthes par Roland Barthes:

Voilà d'un mot, un soir d'octobre 1776 , le sujet "déconstruit" (comme on dit maintenant) : l'ego est bien là, mais c'est pour mieux dire qu'il se quitte, s'expulse de la conscience pleine, se porte au bout de lui-même, là où il se dissout dans le moment : c'est le moment qui est subjectif, individuel, ce n'est pas le sujet, l'individu : thème encore si obscur (tout un devenir devant lui) qu'on le voit travaillé, aujourd'hui, courageusement, par Deleuze. Plus encore : le classicisme entraîne la notation de Rousseau dans une sorte de "sur-avant-garde», fait entendre la musique de quelque chose que nous ne connaissons pas encore : l'abandon de tout paroxysme, l'éloignement de cette violence de langage, que nous croyons "moderne», et qui n'est rien d'autre que le refoulement d'une valeur pourtant bien connue d'autres civilisations (je pense à l'Orient) : l'existence minimale : car «exister» ne se sent pas forcément dans la violence, mais aussi dans ce peu de ciel, d'étoiles, de verdure, qui permit à Rousseau de "partir», c'est-à-dire de raconter. Car voici de nouveau le Récit, et voici de nouveau la question moderne qui nous est posée - ou la contrainte qui nous est rappelée : comment écrire sans ego? C'est ma main qui trace, non celle du voisin. Pour dire que je quitte ce sujet classique, dont je ne veux plus, qui n'est plus possible, je dois le retenir encore un instant, l'instant d'une phrase ${ }^{\mathrm{II}}$.

9. Le Neutre. Cours au Collège de France (1977-1978), texte établi, annoté et présenté par Th. Clerc, Seuil/IMEC, "Traces écrites», 2002, p. 29-30.

Io. Ibid., p. 180-I8I.

II. "D'eux à nous», $O C \mathrm{~V}$, p. 455 . 
Cette belle paraphrase (au sens musical) du texte de Rousseau s'inscrit dans l'évolution des rapports de Barthes et de la "modernité». Quand il découvre que l'ego "n'est plus possible», le lecteur se souvient du quasiaphorisme figurant dans le long article consacré au peintre Réquichot : «Être moderne, c'est savoir ce qui n'est plus possible ${ }^{12}$." La "modernité», on le sait, affectionne les interdits et les formules négatives... Mais le temps où l'on décidait de ce qui est "possible» et de ce qui n'est "plus possible» semble bien terminé pour Barthes. Même s'il n'est pas question de renouer avec une conception "classique" du sujet, c'est-à-dire avec une conception qui ne sait rien de l'inconscient, de l'idéologie ou de l'imaginaire, le ton a changé, les propos diffèrent sensiblement de l'attitude arrogante et assertive de l'avantgarde. Sans renoncer à être moderne, Barthes plaide pour un retour du sujet dont l'ambiguïté de certaines formulations voile la pleine épiphanie. Dans le sillage d'un Rousseau revisité, Barthes renonce à la violence de la modernité, de la modernité comme violence, et dans le prolongement de ce "non-vouloir-saisir ${ }^{13}$ ", c'est une véritable réhabilitation de la subjectivité qu'il nous propose. Il suffit de relire la toute fin du texte pour sentir l'instabilité et le non-dit de la formulation :

Car voici de nouveau le Récit, et voici de nouveau la question moderne qui nous est posée - ou la contrainte qui nous est rappelée : comment écrire sans ego? C'est ma main qui trace, non celle du voisin. Pour dire que je quitte ce sujet classique, dont je ne veux plus, qui n'est plus possible, je dois le retenir encore un instant, l'instant d'une phrase.

Si l'on suit la citation pas à pas, on commence avec une note négative : le Récit, inévitable et indésirable, cette fatalité de toute écriture qu'il convient de retarder aussi longtemps que possible ${ }^{\mathrm{I4}}$. Puis, au regret succède le défi ("comment écrire sans ego?») lancé par la modernité à tout écrivain : il faut absolument éviter de sombrer dans le lyrisme, l'égotisme et tous les pièges d'une affirmation identitaire de sa subjectivité. L'ego apparaît bien encore comme l'ennemi ou du moins comme l'épouvantail à fuir. Mais, la modalité interrogative, en même temps, ne vient-elle pas infléchir le sens? La question ne pourrait-elle pas se lire d'une autre manière, en totale contradiction avec la première interprétation?

L'ambiguïté de la formulation révèle l'ambivalence de Barthes à l'égard de ce fameux ego dont personne ne veut plus. En effet, le «comment écrire...?» n'a pas besoin d'être beaucoup sollicité pour être entendu comme une

I2. «Réquichot et son corps», Euvres complètes, t. IV (1972-1976), nouvelle édition revue, corrigée et présentée par É. Marty, Seuil, 2002, p. 397.

13. La formule apparaît dans Fragments d'un discours amoureux (Seuil, 1977). Elle désigne l'attitude du sujet amoureux qui renonce à exercer le moindre pouvoir à l'égard de l'objet aimé. I4. Le mot «Récit» désigne sans doute toute construction, qu'elle soit narrative ou analytique. 
affirmation que l'on interprétera ainsi : il est impossible d'écrire sans faire une part à l'ego... La suite du texte semble justifier une interprétation qui paraîtrait encore bien hasardeuse. L'interrogation sur le mot "ego» est immédiatement suivie d'une phrase très courte affirmant la singularité de toute main qui écrit et du corps qui la prolonge... D'abord condamné, puis presque justifié, l'ego fait un retour détourné par le biais du mot "corps» qui lui donne une forme acceptable, différente à la fois du sujet cartésien et du fameux "ça parle», cette véritable scie d'une modernité dont Barthes ne veut plus. Entre l'évanescence du "ça» et la consistance du vieil «ego», Barthes refuse de choisir, préférant déplacer la question vers une nouvelle appréhension de la subjectivité qui ne sépare jamais la conscience et le corps, la conscience et le monde. Mais, surtout, grâce au corps, c'est bel et bien le «sujet» comme totalité qui se trouve restauré dans l'ombre honteuse d'un ego qui n'a pas dit son dernier mot... C'est ainsi que le texte se termine sur une belle conclusion indécise où le sens se cherche entre affirmation et rétraction, nécessité et regret, selon une pratique si rusée de l'écriture que le lecteur se demande à quoi s'en tenir exactement. Parti du sentiment de l'existence de Rousseau, de cette forme si particulière de phénoménologie que proposent Les Rêveries $d u$ promeneur solitaire, Barthes, bien loin de "déconstruire» (c'est Deleuze qui est cité, non Derrida), reconstruit plutôt le sujet à tâtons, et de dénégation en interrogation, de rappel en nuance, impose une restauration qui n'est pas loin du lapsus.

\section{La conscience de travail}

La référence rousseauiste éclaire le sens du mot «nature» en l'inscrivant dans une phénoménologie et une éthique fondées sur le désir d'une vie sans «bruits». Mais on ne peut réduire l'attitude de Barthes à cette épochè si particulière, qui n'est plus suspension des savoirs anciens, mais levée des adjectifs, des qualités et des "etc. ", au profit d'une conscience décapée de tout ce qui n'est pas elle. Si l'euphorie du sujet au contact du monde est palpable dans le dernier paragraphe de Roland Barthes par Roland Barthes, un autre mot, venu complexifier la situation, nous éloigne de l'univers de Rousseau et d'une appréhension purement extatique de la conscience dans le monde. Ce mot, on s'en souvient peut-être, est le mot «travail» : "Rien ne rôde, ni le désir, ni l'agression; seul le travail est là, devant moi, comme une sorte d'être universel : tout est plein» ( $R B, \mathrm{p}$. I56). Au cœur d'une description rousseauiste de la nature et du bonheur, le texte de Barthes tire la quête de plénitude et de totalité dans une autre direction. Il ne s'agit plus comme l'enfant 
marocain $^{15}$, dans Incidents, de vivre au ralenti, sur un mode quasi mystique; il s'agit de ménager une place à l'action, de trouver dans l'action, et non plus seulement dans la contemplation, le moyen pour l'homme de s'accorder au monde (c'est la vieille affirmation de tous les humanismes depuis Aristote) et de se protéger de l'Imaginaire (c'est l'obsession barthésienne).

On a parfois décrit Barthes comme un amateur, un dilettante ou un dandy. Les cinq tomes des œuvres complètes, la publication des notes de cours et de séminaires ${ }^{16}$, l'importance des archives encore inexploitées conservées à l'IMEC ${ }^{17}$, l'immense fichier, construction de toute une vie intellectuelle, disent haut et fort que Barthes a beaucoup écrit, beaucoup travaillé et, si l'on fait du travail la clé du bonheur, combien Barthes a connu de moments heureux... Au sens rousseauiste du mot "nature», il convient donc d'ajouter une autre signification, proche du mot "travail», chacun de ces deux mots pouvant passer pour la métaphore de l'autre, grâce à une même obsession de la plénitude ou de la totalité. À la fin de la première section, iconographique, de Roland Barthes par Roland Barthes, sont reproduites trois photographies montrant l'auteur assis à son bureau, dans différents lieux et à des époques différentes. La légende lève toute ambiguïté sur la valeur de cette configuration : «Mon corps n'est libre de tout imaginaire que lorsqu'il retrouve son espace de travail. Cet espace est partout le même, patiemment adapté à la jouissance de peindre, d'écrire, de classer» ( $R B, \mathrm{p} .44-45)$. En devenant acteur, le sujet cesse pour lui d'être un spectacle et un regard, c'està-dire qu'il échappe à l'Imaginaire qui vient à la fois des autres (on me représente) et de soi-même (je me représente). Quand il travaille, le sujet barthésien trouve un parfait équilibre entre une conscience apaisée, héritée de Rousseau, et une conscience active, qui ne déborde pas l'action en train de se faire. Par un miracle sans cesse renouvelable, le travail permet ainsi de trouver la bonne position par rapport au monde et à soi-même, de ménager le bon recul, libérant la pensée tout en neutralisant l'image. Tant qu'il marche devant Eurydice, l'écrivain, tel un nouvel Orphée, ne sait pas qui il est et ce qu'il a fait; tant qu'il travaille, l'écrivain coïncide avec lui-même, s'appréhende comme une totalité heureuse (je suis celui qui écris) et retarde autant

15. «Un gosse assis sur un mur bas, au bord de la route qu'il ne regarde pas - assis comme éternellement, assis pour être assis, sans tergiverser : "Assis paisiblement, sans rien faire, Le printemps vient et l'herbe, croit d'elle-même.» R. Barthes, Incidents, Seuil, 1987.

I6. Les trois cours au Collège de France (Comment vivre ensemble, Le Neutre, La Préparation du roman), le séminaire sur Le Discours amoureux, donné à l'École pratique des hautes études, sont parus au Seuil. Sont en préparation les séminaires sur le Fichier d'auteur et sur Sarrasine...

I7. L'Institut Mémoires de l'édition contemporaine est situé à l'Abbaye d'Ardennes à Caen. 
que possible le moment de se retourner - ce moment du mauvais recul qui constitue l'œuvre en ouvre et le sujet en auteur.

D'une certaine manière, Barthes en a beaucoup rabattu depuis l'optimisme des Mythologies. On se souvient de l'opposition, mise en place dans "Le mythe aujourd'hui», entre le "langage-objet» qui agit sur le monde et le "métalangage» qui se contente de le doubler d'un commentaire analytique ou lyrique. Le premier usage correspond au bûcheron qui transforme la forêt, le second au poète qui chante les arbres, sans avoir prise sur eux. Apparemment condamné au métalangage (il commente le monde), l'intellectuel des années 50 rêve de faire de sa parole un outil qui lui permette d'agir sur la réalité. Vingt ans plus tard, Barthes ne se pose plus avec la même urgence la question de l'engagement. L'opposition entre une parole active et une parole descriptive est à penser dans le cadre nouveau de la lutte contre l'Imaginaire. Il ne s'agit plus d'opposer un langage qui transformerait le monde et un langage qui se contenterait de le décrire. Pour Barthes, désormais, c'est au cœur du seul "métalangage» que tout se joue, comme s'il avait définitivement fait son deuil des formes les plus militantes de l'engagement. À l'opposition, ancienne, du "langage-objet» et du "métalangage» correspond désormais le couple antagoniste de la parole comme travail et de la parole comme image ${ }^{\mathrm{r} 8}$.

La totalité comme plénitude naît donc chez Barthes de ce qu'on appellera "une conscience de travail». Grâce à elle, pour un temps toujours trop bref, le sujet échappe à l'Imaginaire : celui qui travaille n'a pas d'image de lui, il est dans l'action et non dans la représentation. Après Rousseau et son sentiment d'exister, en arriverait-on à Voltaire, l'autre écrivain d'un couple comme les aime beaucoup l'histoire littéraire française (l'article "D'eux à nous " porte sur une comparaison des deux célèbres "philosophes») ? Tout lycéen se souvient de Candide, de sa métairie et de son fameux : «le travail éloigne de nous trois grands maux, l'ennui, le vice, et le besoin». Grâce au travail, tout serait-il donc au mieux dans le meilleur des mondes? Barthes connaît bien ses classiques et le voilà prêt comme un autre à "cultiver son jardin ", dans son bureau à Paris ou à Urt dans sa maison du Pays basque. Mais, en même temps, rien n'est jamais sûr en ce monde et la plénitude comme la totalité sont sans cesse menacées dans leur existence. Et puisque c'est le corps qui définit le nouveau sujet barthésien, c'est un point du corps qui trahit la précarité de ce bonheur si particulier. Voici ce qu'on peut lire dans le fragment "Migraines» :

I8. "Comme Proust malade, menacé par la mort (ou le croyant), nous retrouvons le mot de saint Jean cité, approximativement dans le Contre Sainte-Beuve: "Travaillez pendant que vous avez encore la lumière." ", R. Barthes, «Longtemps, je me suis couché de bonne heure» (OC V, p. 466). 
Lorsque j'ai mal à la tête, ce serait alors comme si j'étais saisi d'un désir partiel, comme si je fétichisais un point précis de mon corps : l'intérieur de ma tête : je serais donc dans un rapport malheureux/amoureux avec mon travail? Une manière de me diviser, de désirer mon travail et d'en avoir peur tout à la fois? («Migraines », RB, p. II3-II4)

Révélée par un mal physique qui affecte la tête, c’est-à-dire le lieu symbolique de la vie intellectuelle, la fragilité de ce bonheur dépend d'une première cause, évidente : on ne peut travailler tout le temps. On sort du travail comme on quitte le rivage du lac ou comme on sort du sommeil : c'est alors le moment de l' "aubade ${ }^{19}$ ", le retour des soucis, des adjectifs et des "etc.».

Au-delà de ce truisme, la migraine ouvre sur une réalité beaucoup plus délicate, liée à l'hyperactivité de la conscience chez le sujet Barthes. Quand Rousseau s'enchante d'une subjectivité qui habite le présent avec légèreté, Barthes parce qu'il aime le travail révèle en lui l'existence d'une conscience perpétuellement en éveil, moins engoncée dans la chaude matérialité du corps que celle du promeneur bercé par le lac de Bienne. Proche en cela de Valéry, héritier d'une tradition française faite d'autoanalyse et de lucidité triomphantes, Barthes a beaucoup de mal à se laisser aller - d'où la fascination qu'exerce sur lui le modèle rousseauiste ou le modèle oriental d'une "existence minimale». La migraine et son malaise témoignent de l'obsession d'un homme de tête qui n'oublie jamais de se poser la question du sens ("Qu'est-ce que ça veut dire», $R B$, p. I33), qui existe constamment sur deux plans complémentaires, l'action et le regard sur l'action, au point de vivre cette situation comme une «malédiction»:

Et cependant : au niveau de son corps, sa tête ne s'embrouille jamais. C'en est une malédiction : aucun état flou, perdu, second : toujours la conscience : exclu de la drogue et cependant la rêvant : rêvant de pouvoir s'enivrer (au lieu d'être tout de suite malade); escomptant autrefois d'une opération chirurgicale au moins une fois dans sa vie une absence, qui lui fut refusée, faute d'une anesthésie générale. ("Ma tête s'embrouille», $R B$, p. 153)

Fondé sur une définition très active et très intellectuelle de la conscience, le bonheur lié au travail se voit remis en cause par cette hyperactivité même qui ne laisse ni répit ni repos. Bien plus, en instaurant le sujet comme spectateur de lui-même, la conscience crée une forme de recul propice à la création et à la lucidité; mais elle risque, également, de privilégier le spectacle sur l'action, l'image du travail sur le travail et de rétablir l'Imaginaire au cœur même du processus qui tentait de la contenir. Quand on écrit, en effet, même si la tâche est suffisamment absorbante pour éviter d'avoir à se retourner sur soi-

19. Voir «Réveil», l'enseigne de la figure "L'aubade» dans Fragments d'un discours amoureux, op. cit., p. 24I : «RÉVEIL. Modes divers sous lesquels le sujet amoureux se retrouve, au réveil, réinvesti par le souci de sa passion.» 
même et de découvrir l'évanouissement d'Eurydice, le recul propre à toute activité intellectuelle, le caractère impérieux de l'hyperconscience risquent à tout moment de s'appliquer au sujet lui-même, transformé, malgré qu'il en ait, en spectacle, c'est-à-dire en image sagement incluse dans un système imaginaire. Quand il déclare, par exemple, «J'écris classique», Barthes ne peut éviter le retour de l'adjectif (ici, «classique»), offrant une représentation de lui à la contemplation du lecteur. Toute conscience de travail emportée par le recul qui la caractérise se voit menacer de devenir une conscience $d u$ travail et par là même de mettre fin au bonheur d'une lucidité insouciante.

Lecteur de Valéry (la Jeune Parque se «voit voir»), mais aussi de Lacan, Barthes se refuse à être dupe de sa propre clairvoyance. Le commentaire de deux photos du Roland Barthes par Roland Barthes semble entrer dans un dialogue secret avec un passage des Quatre Concepts fondamentaux de la psychanalyse, le séminaire de Lacan auquel il est souvent fait référence dans $L e$ Discours amoureux. Contemplant son visage reproduit à deux époques différentes, Barthes s'interroge de la façon suivante :

Mais je n'ai jamais ressemblé à cela! - Comment le savez-vous? Qu'est-ce que ce "vous" auquel vous ressembleriez ou ne ressembleriez pas? Où le prendre? À quel étalon morphologique ou expressif? Où est votre corps de vérité? ( $R B$, p. 42)

Puis, après ce questionnement portant sur l'impossible connaissance de soi, Barthes aborde la question du regard pour en arriver à l'aporie du fameux «voir voir»:

Vous êtes le seul à ne jamais vous voir qu'en image, vous ne voyez jamais vos yeux, sinon abêtis par le regard qu'ils posent sur le miroir ou sur l'objectif (il m'intéresserait seulement de voir mes yeux quand ils te regardent) : même et surtout pour votre corps, vous êtes condamné à l'imaginaire. ( $R B$, p. 42)

Dans le lointain - très lointain sillage - de l'interrogation lacanienne sur "l'œil et le regard ${ }^{20}$ ", Barthes sait prendre ses distances à l'égard de la tradition phénoménologique...

20. «Nul besoin de nous reporter à je ne sais quelle supposition de l'existence d'un voyant universel. Si la fonction de la tache est reconnue dans son autonomie et identifiée à celle du regard, nous pouvons en chercher la menée, le fil, la trace, à tous les étages de la constitution du monde dans le champ scopique. On s'apercevra alors que la fonction de la tache et du regard y est à la fois ce qui commande le plus secrètement, et ce qui échappe toujours à la saisie de cette forme de la vision qui se satisfait d'elle-même en s'imaginant comme conscience.

Ce en quoi la conscience peut se retourner sur elle-même - se saisir, telle la Jeune Parque de Valéry, comme se voyant voir - représente un escamotage. Un évitement s'y opère de la fonction du regard..." J. Lacan, Le Séminaire Livre XI, Les Quatre Concepts fondamentaux de la psychanalyse, texte établi par J.-A. Miller, Seuil, «Le Champ freudien», 1973, p. 7I. 


\section{La « distance convenable»}

C'est donc bien la question de la distance qui se pose : à quelle distance fautil se tenir de soi-même et du monde pour agir sans transformer les choses en spectacle desséché et le sujet en "frite ${ }^{21}$ " livrée à la logosphère? Une fois n'est pas coutume, la leçon vient des porcs-épics. Réfléchissant à la situation du sujet amoureux par rapport à l'objet de son désir, Barthes revient sur une parabole de Schopenhauer reprise par Freud dans ses Essais de psychanalyse:

[...] les porcs-épics, souffrant du froid, se rapprochaient alors les uns les autres, se piquant, s'éloignant et reconnaissant le processus cyclique : «ces alternatives de rapprochement et d'éloignement durèrent jusqu'à ce qu'ils aient trouvé une distance convenable ${ }^{22}$ où ils se sentirent à l'abri des maux [...]" (la difficulté : distance convenable: tautologie improductive $)^{23}$.

Comment se tenir à l'abri des maux quand on est un porc-épic (mais la réponse ne nous intéresse pas vraiment)? comment se situer dans l'espace social quand on est un amoureux ou tout individu confronté à la question éthique? Et pour revenir à notre propos, comment la conscience de travail réussira-t-elle à préserver l'équilibre entre recul et proximité? Au fond, c'est toujours la même question formulée sur tous les tons, selon les occasions ou les circonstances : à quelle distance faut-il se tenir des autres et de soi-même pour éviter de sombrer dans la coalescence (je colle à l'image et sombre dans l'Imaginaire) ou dans un recul excessif (je me distancie tellement que je me transforme en spectacle, c'est-à-dire, une fois encore, en objet de l'Imaginaire)?

2I. Voir R. Barthes, "L'image» (OC V, p. 516-517) : "Combat des systèmes de langage : métaphore de la ventouse. Revenons maintenant au combat des Images («image» : ce que je crois que l'autre pense de moi); comment une image de moi "prend"-elle au point que j'en sois blessé? Voici une nouvelle métaphore : "Dans la poêle, l'huile est étalée, plane, lisse, insonore (à peine quelques vapeurs) : sorte de materia prima. Jetez-y un bout de pomme de terre : c'est comme un appât lancé à des bêtes qui dormaient d'un œil, guettaient. Toutes se précipitent, entourent, attaquent en bruissant; c'est un banquet vorace. La parcelle de pomme de terre est cernée - non détruite, mais durcie, rissolée, caramélisée; cela devient un objet : une frite." Ainsi, sur tout objet, le bon système langagier fonctionne, s'affaire, cerne, bruit, durcit et dore. Tous les langages sont des micro-systèmes d'ébullition, des fritures. Voilà l'enjeu de la Machè langagière. Le langage (des autres) me transforme en image, comme la pomme de terre brute est transformée en frite.»

22. Dans Comment vivre ensemble, son premier cours au Collège de France, Roland Barthes bâtit sa recherche à partir d'un fantasme, que cristallise le mot «idiorrythmie». Ce mot, qui désigne le mode de vie de moines rattachés à un monastère mais vivant en marge de la communauté, renvoie à toutes les formes de sociabilité qui tentent d'harmonisent vie collective et vie individuelle (Comment vivre ensemble Cours et séminaires au Collège de France (19761977), texte établi, annoté et présenté par Cl. Coste, Seuil/IMEC, "Traces écrites», 2002).

23. Le Discours amoureux, op. cit. p. 452. 
On est bel et bien confronté à la quadrature du cercle : quand on cherche à décrire la conscience par le biais très banal d'une métaphore spatiale, on est rapidement soumis à une insoluble contradiction. Parce que la conscience n'existe pas sans objet, parce que le sujet rousseauiste et barthésien investit tel ou tel aspect de la nature (le ciel, une branche) au point de se confondre avec lui et de concevoir la plénitude comme parfaite adhésion, la proximité appartient de plein droit à l'exercice de la conscience; inversement, parce qu'il n'y a pas de conscience sans recul, la distance entre également de plein droit dans la constitution d'une bonne relation au monde. Il ne s'agit donc plus de choisir la distance contre la proximité ou de faire le choix inverse, mais de trouver le bon dosage qui permet d'assurer un équilibre quasi impossible. Un terme, la "fascination", exprime à lui tout seul cet alliage de deux positions que le sens courant rend inconciliables. Qu' est-ce que la fascination en effet sinon cette relation paradoxale qui me tient collé à un objet dont je me tiens à distance? Au fond, être fasciné (tant pis pour le paradoxe facile), c'est adhérer de loin...

Mais le terme de "fascination", souvent associé à la bêtise de l'amoureux, ne prend que rarement en charge le rêve d'un équilibre entre deux positions perçues comme incompatibles. Il faudra trouver d'autres mots pour défendre le même projet : sous la plume de Barthes, ce sera «méditation» qui concilie bien l'intériorité et l'action, ou "sidération" qui, dans le souvenir lointain de son étymologie, porte en lui tout un ciel étoilé. À l'opposé donc de la "coalescence», de la "colle» ou de l'«analogie», la plupart du temps connotées négativement par Barthes parce qu'elles livrent le sujet pieds et poings liés à l'Imaginaire, le mot "méditation ", à défaut d'une solution, offre le réconfort d'une verbalisation. Dans le texte liminaire de Roland Barthes par Roland Barthes, l'auteur justifie ainsi le choix des photos qui ouvrent le volume :

Lorsque la méditation (la sidération) constitue l'image en être détaché, lorsqu'elle en fait l'objet d'une jouissance immédiate, elle n'a plus rien à voir avec la réflexion, fût-elle rêveuse, d'une identité; elle se tourmente et s'enchante d'une vision qui n'est nullement morphologique (je ne me ressemble jamais), mais plutôt organique. $(R B, \mathrm{p} .7)$

Cette citation extraordinairement dense brasse dans une seule phrase une multitude de termes apparemment contradictoires, renvoyant à la distance («détaché», «objet», "vision»...), à la proximité temporelle ou spatiale («immédiate», «jouissance»...), ménageant à la fois la présence du sujet physiologique ("organique») et le refus de vieil ego classique («identité»).

Hésitant à choisir un des deux termes de l'oxymore (proximité distante), Barthes valorise finalement la distance, tant le vocabulaire de la proximité parait péjoratif dans son univers intellectuel. Quitte à donner un peu le 
tournis, ce sera donc sur la distance que se fondera un humanisme un peu naif, opposant clairement l'homme et le taureau :

(Le taureau voit rouge lorsque son leurre lui tombe sous le nez; les deux rouges coïncident, celui de la colère et celui de la cape : le taureau est en pleine analogie, c'est-à-dire en plein imaginaire. Lorsque je résiste à l'analogie, c'est en fait à l'imaginaire que je résiste : à savoir : la coalescence du signe, la similitude du signifiant et du signifié, l'homéomorphisme des images, le Miroir, le leurre captivant. Toutes les explications scientifiques qui ont recours à l'analogie - et elles sont légion - participent du leurre, elles forment l'imaginaire de la Science.)» ("Le démon de l'analogie», RB, p. 50)

Mais, dans ce bestiaire philosophique, la parabole des porcs-épics ne donnaitelle pas déjà la réponse, indiquant clairement de quel côté penchait le choix de Barthes pour sortir de l'impasse? Comme on s'en souvient, il s'agissait bel et bien de trouver, non pas la proximité, mais la «distance convenable», de définir le jeu qui relâche et qui préserve le lien avec le monde et avec les autres.

Dans Roland Barthes par Roland Barthes, la même idée revient sans cesse, reformulée de manière différente : si la bêtise coïncide avec la colle, si Bouvard et Pécuchet finissent par se transformer en copistes, il convient de miser à fond sur le pouvoir de déprise qui caractérise la conscience et la pensée, sur une pratique du dédoublement qui permet d'échapper à la coalescence. Les formules ne manquent pas : "le sujet dédoublé (ou s'imaginant tel) parvient parfois à signer son imaginaire» ("L'imaginaire», $R B$, p. 99); ou encore : "Il suffirait de se voir bête pour l'être moins» («Bête», $R B$, p. IO2). Mais le ton, on en conviendra, n'est guère à l'enthousiasme... Si une pratique trop confiante de la distance ouvre directement sur l'Imaginaire, Barthes sait bien qu'il ne lui reste plus qu'à entrer dans l'espace du jeu, qu'à se confronter au simulacre, qu'à prendre ses distances avec la distance... Ce qui revient à faire la part du théâtre, pour ne pas être dupe de sa propre intelligence. Ainsi, Barthes va chercher à théâtraliser la conscience créatrice - ce qu'il fait dans $L a$ Chambre claire (en reproduisant pour son lecteur les errances et les impasses de sa recherche) et, plus hyperboliquement encore, dans son dernier cours au Collège de France. De quoi s'agit-il en effet dans La Préparation du roman? Longtemps hostile au roman, puis fasciné par un genre qu'il ne sait pas trop comment aborder, Barthes décide de consacrer sa recherche à la conscience créatrice du romancier, dont il restitue pas à pas les différentes étapes. Au délire de la Jeune Parque, le professeur préfère la démarche plus ironique, plus hésitante de celui qui fait semblant de faire pour mieux comprendre, à la fois, les secrets de la forme et le fonctionnement de sa propre créativité. Articulant citations et références empruntées aux grands écrivains (Flaubert, 
Proust...), mêlant à l'ensemble son propre regard et sa propre expérience, Barthes se met dans la position de quelqu'un qui va écrire un roman. De cette théâtralisation de la conscience, Barthes libère une double énergie : celle du professeur qui fait cours sur un fantasme obsessionnel et celle de l'auteur qui écrit son dernier livre - cette Chambre claire qu'on peut lire comme une lointaine version d'À la Recherche du temps perdu...

\section{Écoute $^{24}$}

Mais le théâtre ne va pas sans danger. En jouant efficacement avec les décrochements de conscience, il permet de mettre en scène la distance et d'éviter d'en être dupe. En même temps, précisément parce qu'il est spectacle, le théâtre n'est pas loin de l'hystérie. Menaçante comme la bêtise, l'hystérie se produit toutes les fois que "je» me donne en spectacle, préparant le retour d'un Imaginaire triomphant. Une fois encore, que faire avec ce regard qui porte en lui la mort, comme Méduse ou Orphée? Entre réalité et métaphore, on s'efforcera de l'alléger, de rendre la perception plus abstraite, moins figurative, comme nous le raconte Barthes à propos d'une conversation avec sa boulangère :

Le temps qu'il fait : Ce matin, la boulangère me dit : il fait encore beau! mais chaud trop longtemps! (Les gens d'ici trouvent toujours qu'il fait trop beau, trop chaud). J'ajoute : et la lumière est si belle! Mais la boulangère ne répond pas, et une fois de plus j'observe ce court-circuit du langage, dont les conversations les plus futiles sont l'occasion sûre; je comprends que voir la lumière relève d'une sensibilité de classe; ou plutôt, puisqu'il y a des lumières "pittoresques» qui sont certainement goûtées par la boulangère, ce qui est socialement marqué, c'est la vue "vague», la vue sans contours, sans objet, sans figuration, la vue d'une transparence, la vue d'une non-vue (cette valeur infigurative qu'il y a dans la bonne peinture et qu'il n'y a pas dans la mauvaise). En somme, rien de plus culturel que l'atmosphère, rien de plus idéologique que le temps qu'il fait. («Le temps qu'il fait ", $R B$, p. 153 )

Dans cet épisode emprunté au petit théâtre de la vie quotidienne, on retrouve, bien sûr, un thème récurrent de toute l'œuvre de Barthes : le malentendu, le dialogue de sourds, la division des langages, la différence culturelle vécue comme une épreuve; mais pour qui s'intéresse au regard, l'essentiel n'est pas là. Ce que nous offre Barthes en décrivant «la lumière du SudOuest", c'est une belle expérience scopique où se conjuguent la sensualité et l'immatérialité... Voir une "transparence», voir une "non-vue», telles sont les sorties que propose Barthes pour échapper à la tyrannie du mauvais œil.

24. Sur cette question, voir R. Barthes, «Écoute», OC V, p. 340. 
Mais cela suffit-il? Afin de sauver la plénitude d'un sujet qui trouve une forme de totalité dans le travail et l'action, il faut aller plus loin, ne pas se contenter de dématérialiser le spectacle.

De l'œil, Barthes glissera donc vers l'oreille; du regard, il passera à l'écoute comme moyen d'instaurer une autre forme de "coïncidence» avec le monde. Un fragment de Roland Barthes par Roland Barthes propose une expérience de «coïncidence» qui repose entièrement sur l'écoute et le piano :

Je m'enregistre jouant au piano; au départ, c'est par curiosité de m'entendre; mais très vite je ne m'entends plus; ce que j'entends, c'est, quelque apparence de prétention qu'il y ait à le dire, l'être-là de Bach et de Schumann, la matérialité pure de leur musique; parce qu'il s'agit de mon énonciation, le prédicat perd toute pertinence; en revanche, fait paradoxal, si j'écoute Richter et Horowitz, mille adjectifs me viennent : je les entends, eux, et non pas Bach et Schumann. - Que se passe-t-il donc? Lorsque je m'écoute ayant joué - passé un premier moment de lucidité où je perçois une à une les fautes que j'ai faites -, il se produit une sorte de coïncidence rare : le passé de mon jeu coïncide avec le présent de mon écoute, et dans cette coïncidence s'abolit le commentaire : il ne reste plus que la musique (il va de soi que ce qui reste, ce n'est nullement la «vérité» du texte, comme si j'avais retrouvé le «vrai» Schumann ou le "vrai «Bach). («La coïncidence», $R B, \mathrm{p} .59$ )

Renouant avec la plénitude, éprouvant la sensation de ne faire plus qu'un Tout, Barthes connait dans cette page une double coïncidence dont l'écoute est la clé. La première est implicite : évoquant sa propre pratique instrumentale, le pianiste se situe d'emblée hors de portée de l'Imaginaire. Entièrement absorbé et défini par son jeu, Barthes ne se représente pas, échappe à toute forme de regard qui le figerait dans une posture. Mais ce n'est pas tout. Sur cette première coïncidence, s'en greffe une seconde, explicitement et longuement développée par le texte. S'écoutant jouer du piano, Barthes abolit la différence entre le jeu et l'écoute, entre la création et la réception. À une première totalité liée au présent du jeu (le sujet coïncide avec lui-même dans l'interprétation) s'en ajoute une seconde : passé et présent, associés dans une même totalité, abolissent momentanément le temps, créant une fragile transcendance qui rappelle bien évidemment, mais en mineur, la révélation du Temps retrouvé.

Pourtant, si la musique, entre réalité et métaphore, se donne comme le meilleur moyen d'échapper au théâtre et à la tyrannie du regard, tout est réversible dans le monde de Barthes : l'écoute elle aussi connaît son errance, ses dangers et son malaise. Évoquant la figure de «Méduse», c'est-à-dire le mauvais regard, un fragment de Roland Barthes par Roland Barthes dérive d'une manière inattendue du côté de l'oreille. C'est comme si, du Temps retrouvé, l'expérience subjective régressait vers cet étrange voyeurisme sonore que décrit le narrateur au début de Sodome et Gomorrhe II quand il épie la rencontre de Charlus et Jupien : 
Méduse, ou l'Araignée, c'est la castration. Elle me sidère. La sidération est produite par une scène que j'écoute mais ne vois pas : mon écoute est frustrée de sa vision : je reste derrière la porte.

La Doxa parle, je l'entends, mais je ne suis pas dans son espace. Homme du paradoxe, comme tout écrivain, je suis derrière la porte; je voudrais bien la passer, je voudrais bien voir ce qui est dit, participer moi aussi à la scène communautaire; je suis sans cesse à l'écoute de ce dont je suis exclu; je suis en état de sidération, frappé, coupé de la popularité du langage. ("Méduse», $R B$, p. II2)

Cette fois-ci, les mots et les valeurs sont inversés : la «sidération» ne vient plus au secours d'une méditation heureuse sur la présence organique du sujet, l'écoute suscite l'inquiétude, la distance critique est perçue comme une forme de déréliction... Creusant une distance non "convenable», l'écoute retrouve une autre maladie, celle du regard précisément, quand il fonctionne en solitaire, à la pointe du paradoxe :

J'ai une maladie : je vois le langage. Ce que je devrais simplement écouter, une drôle de pulsion, perverse en ce que le désir s'y trompe d'objet, me le révèle comme une "vision", analogue (toutes proportions gardées!) à celle que Scipion eut en songe des sphères musicales du monde. À la scène primitive, où j'écoute sans voir, succède une scène perverse, où j'imagine voir ce que j'écoute. L'écoute dérive en scopie : du langage, je me sens visionnaire et voyeur. ("Je vois le langage», $R B$, p. I4I)

Les deux expériences sont parfaitement symétriques : l'écoute du voyeur et la vision du langage indiquent très clairement que la solution n'appartient à aucun type de perception en particulier. Mais la dérive de chacun des deux sens l'un vers l'autre ne dessine-t-elle pas une porte de sortie? Quand toutes les issues semblent barrées, quand «mon écoute est frustrée de sa vision" et que "l'écoute dérive en scopie», il suffit sans doute de pousser un peu plus loin le jeu des synesthésies.

Même s'il ne le dit pas explicitement, Barthes ajoute une nouvelle coïncidence aux coïncidences, rémunère la défaillance de chaque mode de perception en jouant sur la rencontre de l'écoute et de l'ouïe. En faisant coïncider l'œil et l'oreille, en les associant dans un souci quasi prophylactique, Barthes crée une nouvelle totalité et se définit ainsi comme écrivain. En effet, si la main trace les signes pour celui qui lira le texte comme un spectacle, la voix n'est jamais absente de l'écriture, qu'il s'agisse de l'auteur ou du lecteur. C'est pourquoi la présence de l'écoute et du monde sonore, par la force impérieuse de la métaphore, nous rappelle que la littérature a tout à gagner en se situant dans le sillage de la musique ${ }^{25}$. Écrire en écoutant, écouter en lisant, se

25. "J'attends donc, peut-être, une transformation de l'Écoute - et peut-être me viendrat-elle, sans métaphore, par la musique, que j'aime tant." La Préparation du roman, op. cit., p. 384 . 
montrer sensible dans la graphie des mots à la mélodie du sujet créateur, c'est sans doute le meilleur moyen de trouver cette "distance convenable» qui installe la bonne proximité à l'égard du monde et des autres. La musique, comme on pouvait s'y attendre, renvoie chez Barthes à l'harmonie de la structure, à la suspension du sens (la signifiance), à la sociabilité retrou$v^{2} e^{26} .$. Mais d'une manière plus personnelle, la musique vaut aussi chez lui comme modèle de coïncidence heureuse entre le sujet et l'action, la création et la réception, la conscience et le présent du monde...

Le démon de la totalité n'a jamais abandonné Barthes : la solution est tantôt rousseauiste, du côté d'une vie minimale et d'une subjectivité sans histoire; tantôt du côté de l'action et du travail, seuls capables d'affronter le temps qui dure et l'échelle de la vie, seuls capables de suspendre, durant la marche, le retour des images et de l'Imaginaire. Or, transcendance des transcendances, c'est précisément la musique qui permet de dépasser et d'unifier toutes ces totalités. Active et contemplative, subjective et collective, la musique vaut comme l'utopie de l'écrivain. C'est bien pour cela, comme le précise Barthes dans La Préparation du roman ${ }^{27}$ qu'il «faut toujours penser l'Écriture en termes de musique». C'est bien pour cela que la musique, l'amour et la conscience entretiennent une relation secrète sous le regard protecteur de la littérature. En quête de totalité et d'euphorie, l'amoureux qui cherche l'harmonie d'un monde "inouï», qui se met à l'écoute de la nouveauté (inaudita), se révèle comme le seul capable de métamorphoser le désir de comprendre et la vie de la pensée. À la conscience amoureuse, donc, de servir de guide à la conscience de l'écrivain :

Je veux changer de système : ne plus démasquer, ne plus interpréter, mais faire de la conscience une drogue, et par elle accéder à la vision sans reste du réel, au grand rêve clair, à l'amour prophétique.

(Et si la conscience - une telle conscience - était notre avenir humain? Si, par un tour supplémentaire de la spirale, un jour, éblouissant entre tous, toute idéologie réactive disparue, la conscience devenant enfin ceci : l'abolition du manifeste et du latent, de l'apparence et du caché? S'il était demandé à l'analyse non pas de détruire la force (pas même de la corriger et de la diriger), mais seulement de la décorer, en artiste? Imaginons que la science des lapsus découvre un jour son propre lapsus, et que ce lapsus soit : une forme nouvelle, inouïe, de la conscience ${ }^{28}$ )

26. «La musique de quelque chose que nous ne connaissons pas encore : l'abandon de tout paroxysme», "D'eux à nous", op. cit.

27. Op. cit., p. 32I.

28. Fragments d'un discours amoureux, op. cit., p. 72-73. 